\title{
Minimization of the Arrestment Error in One-Pan, Two-knife Balance Systems
}

\author{
H. A. Bowman and H. E. Almer
}

(May 1, 1963)

\begin{abstract}
The arrestment error is defined as the error associated with the arrestment, unloading, reloading, and release cycle after correction for balance drift and changes in sensitivity with beam tilt. In a well-designed one-pan, two-knife, constant-load balance the observable arrestment error is caused by inconstancy of the perpendicular distance from the effective point of suspension of the hangdown to the effective axis of rotation of the center knife. Four causes for this distance to vary are recognized and their effective removal discussed. The requirements for two arrestment systems are then set out, one in which the balance bearings remain in contact indefinitely under nearly constant load, the other in which the bearings are uncoupled in such a way as to permit the bearings to be recoupled in a contact configuration as nearly identical to the previous configuration as possible.

A 1-kg test instrument is described with which many balance systems can be experimentally investigated including the two arrestment systems mentioned above (the "constantload" and "rocking-beam" systems).

The arrestment error (expressed as a standard deviation) for the "rocking-beam" system can be reduced to $1.5 \times 10^{-8}$ of the load; for the "constant-load" system to $3 \times 10^{-9}$. When slight external vibrational disturbances are not scrupulously excluded occasional unpredictable shifts in balance indication are observed. These exceed the normal arrestment errors by about an order of magnitude. The arrestment error does not vary greatly with beam-swing period over its normal range.
\end{abstract}

\section{Introduction}

This paper is in a series describing studies on sources and possible elimination of residual weighing errors on balances for weight calibrations operating near the highest attainable precision. Previous studies were concerned with humidity of the ambient air [1], ${ }^{1}$ with oscillation of the pan assembly [2], and with the weighing series used [3]. The present paper describes studies on the arrestment error in one-pan, two-knife constant-load balances, whose suitability for weight calibration has been previously advocated by Peiser [4]. A generalpurpose performance test for such balances has also been published [5].

The arrestment systems of high-precision balances usually involve complex parts, they are the most difficult to adjust, and have long been recognized as an important, if not always the dominating source of residual weighing errors.

In sections 2 and 3 we will describe and define terms relating to arrestment systems and errors which are brought into relation with the equilibrium conditions of a balance with fixed counterweight. In section 4 we discuss the principal residual sources of arrestment errors in balances of high precision operated expertly. For each of these errors one or more possible cures are suggested. In section 5 a test instrument is described on which several kinds of arrestments can be tried and evaluated. The experimental results are then given in section 6 .

\footnotetext{
1 Figures in brackets indicate the literature references at the end of this paper.
}

\section{Conventional Arrestment and Release}

Figure 1 shows schematically the essential parts of the oscillating structure of a simple one-pan, two-

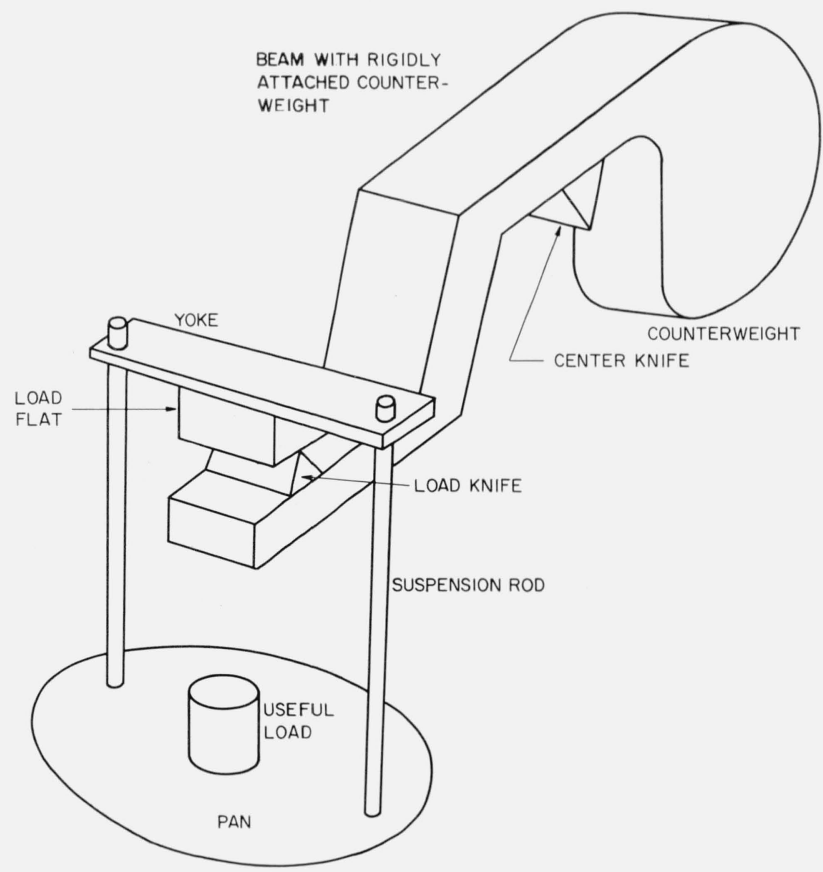

FIGURE 1. Schematic sketch of the oscillating structure of the basic form of the one-pan, two-knife balance.

The counterweight is made of such a mass and placed at such a distance that the balance is "on-scale" when the design load is placed on the pan. 
knife substitution balance. Unloading and reloading of the pan is potentially the most damaging process in a weighing cycle. The suspension, for example, designed to accept vertical loads, may have horizontal forces impressed upon it. The arrestment system is designed to protect balances during this reloading process.

We define an arrested balance as one placed in a condition for unloading and reloading with minimum disturbance to the balance adjustment. We define a released balance as one in which the beam and hangdown can oscillate about the knife-bearings free from constraints other than those imposed by the gravitational field, the surrounding atmosphere, and - if used - a beam-swing damping system.

The arrestment system is designed to arrest as well as to release the balance with the minimum disturbance to that balance adjustment. Conventionally the system performs the following tasks:

1. It removes the freedom of swing from the beam.

2. It removes the freedom of swing from the pan and suspension assembly (hangdown).

3. It firmly supports the hangdown so that the load flat is no longer in contact with the load knife and so that loads can be placed on and/or removed from the pan.

4. It lifts the beam so that the center knife is out of contact with its flat.

5. It is able to reverse steps 1 to 4 recoupling both knives and flats in precisely the original contact configuration and releasing the pan without appreciable swing.

Steps 1 to 4 are called arrestment, step 5 release.

\section{The Arrestment Error}

Corwin [6] has previously presented a discussion of the arrestment error. Although our analysis differs in detail, our ideas are based on his. The arrestment error is here defined as the sum of all errors associated with the "operating cycle"- that is arrestment, unloading, reloading, and release. This arrestment error is readily measured experimentally by change of balance indication upon repeated reloadings of the same weight. Random variability of the mass of the test weight during the operating cycle would make the observed arrestment error too large. If the transfer mechanism offers one weight to the pan more reproducibly than two different weights, the observed arrestment error would be too small. We believe both of these effects are negligible in our experiments. Slow balance drift and changes of sensitivity with tilt are separately evaluated and excluded from the experimentally determined arrestment error.

The inclusion of errors from unloading and reloading in our definition of arrestment error is an important innovation. It is true that loading and unloading are not part of the arrestment operation. Experimental observations, however, of the errors due to arrestment and release alone give observed errors that are too low to be significant from the view point of a real weighing cycle. The significant test of an arrestment system is how well it protects the reproductibility of a balance during the unloading and reloading operation.

The idealized equilibrium conditions of a released one-pan two-knife balance is:

$$
M r \cos \theta+M_{\beta} p \cos \phi=0,
$$

where $M$ is the total mass of the hangdown whose effective point of suspension is at a perpendicular distance $r$ from the effective center-knife axis. The line parallel to the distance $r$ is inclined by $90+\theta$ to the gravitational-field direction and $M_{\beta}$ is the mass of the beam with its rigidly attached counterweight, the combined center of gravity of which is at a perpendicular distance $p$ from the effective center-knife axis. The line parallel to the distance $p$ is inclined by $90+\phi$ to the gravitational field.

$\theta+\phi$ is a constant of the balance related to the readout angle by another constant.

In a well-designed beam, to be discussed in a later paper, $p$ and $\phi$ will remain constant during a weighing cycle to well within the weighing precision. The masses $M$ and $M_{\beta}$ will also remain constant to higher precision. Variability of $\theta$ before and after the arrestment cycle must be caused almost exclusively by changes in $r$. This realization simplifies the search for possible arrestment errors. One further consideration may also be useful. As has been previously pointed out [6] one source of variability in $r$ arises from nonequality and nonuniformity of knife-edge radii. Whereas load-dependence of these imperfections are eliminated as possible sources of weighing error in constant-load balances, $\theta$-dependent variations remain. Balances should therefore be used as zero-point instruments or calibrated for systematic variations with $\theta$. Arrestment errors systematic with the observed quantity, $\theta$, need not be investigated; it should be noted that they are excluded by the definition given in section 2 .

\section{Sources and Elimination of Arrestment Errors}

\subsection{Nonparallelism of Knives}

The schematic diagrams (fig. 2) show how the arm length $r$, the perpendicular distance of the effective point of suspension (eps) from the effective axis of roatation of the center knife, is changed by nonparallel knives when associated with imperfect reproducibility of centering of load along a line parallel to the terminal knife.

The parallelism of knives is a most important balance adjustment which should always be carried out to the highest attainable perfection. Nevertheless the remaining nonparallelism in practice is a demonstrable source of weighing imprecision. Although all too often forgotten this error was recognized long ago by Thiessen [7] and corrected by insertion of an intermediate knife edge perpendicular and coplanar with the terminal knife as illustrated in figure 3 . The finite curvature of the intermediate knife will permit a very small 

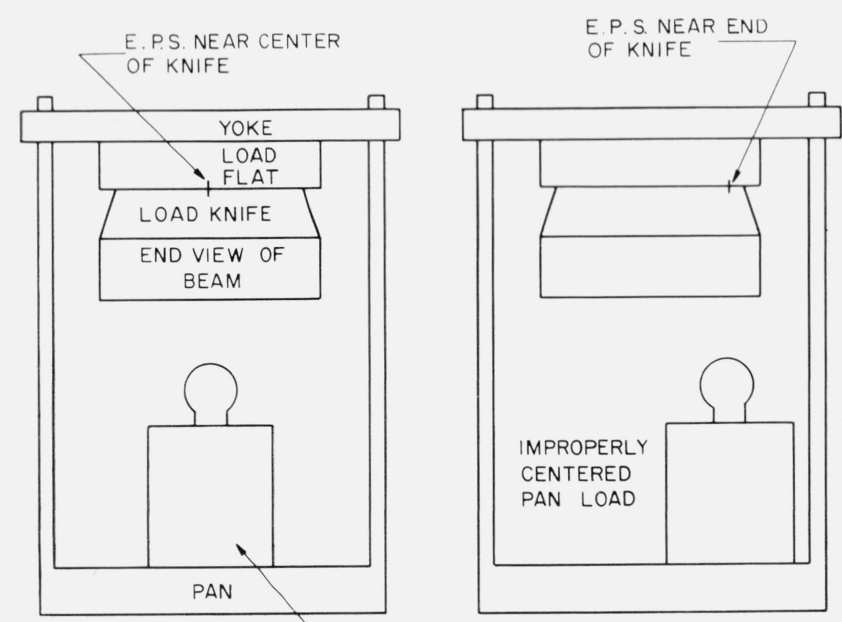

PROPERLY CENTERED

PAN LOAD

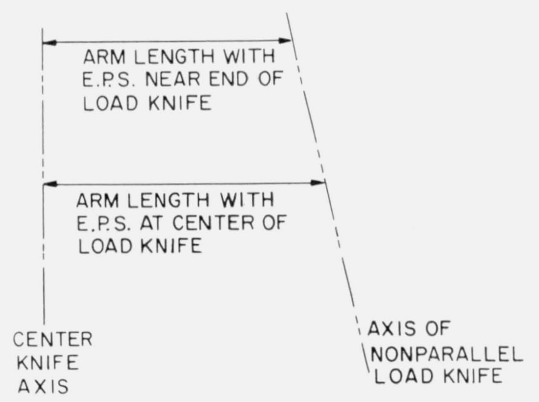

FiguRE 2. The upper two diagrams indicate how the effective point of support (the eps) of the suspension by the beam is caused to migrate along the load knife edge due to failure to assure proper centering of the load on the pan.

It the bottom is a sketch showing how the arm length, $r$, varies when the two knives are not parallel under conditions of eps migration.

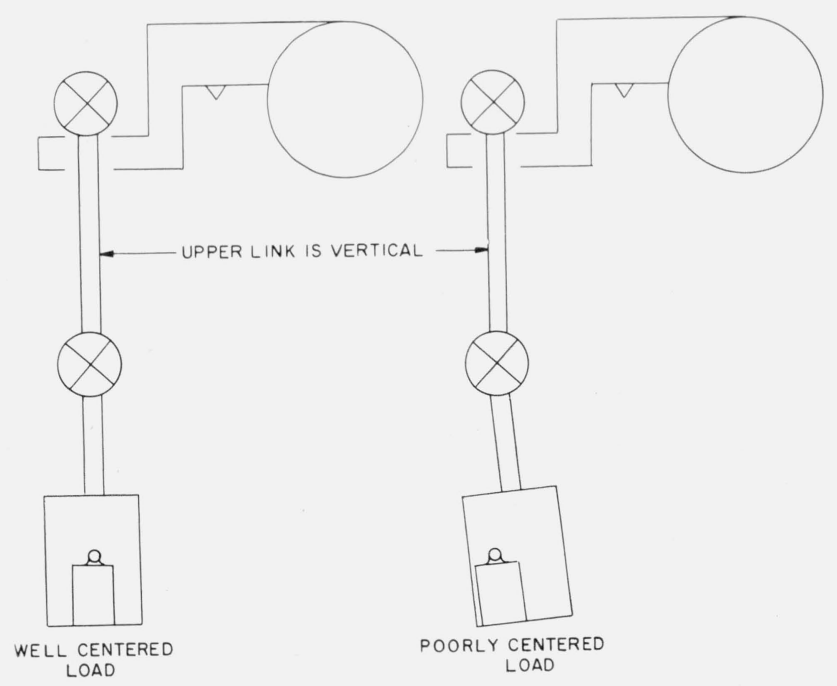

Figure 4. This sketch illustrates the principle that the link connecting two universal couplings will hang at a constant angle with respect to vertical.

The upper universal coupling is the knife-edge compensator and the lower is a ball bearing gimbal ring.

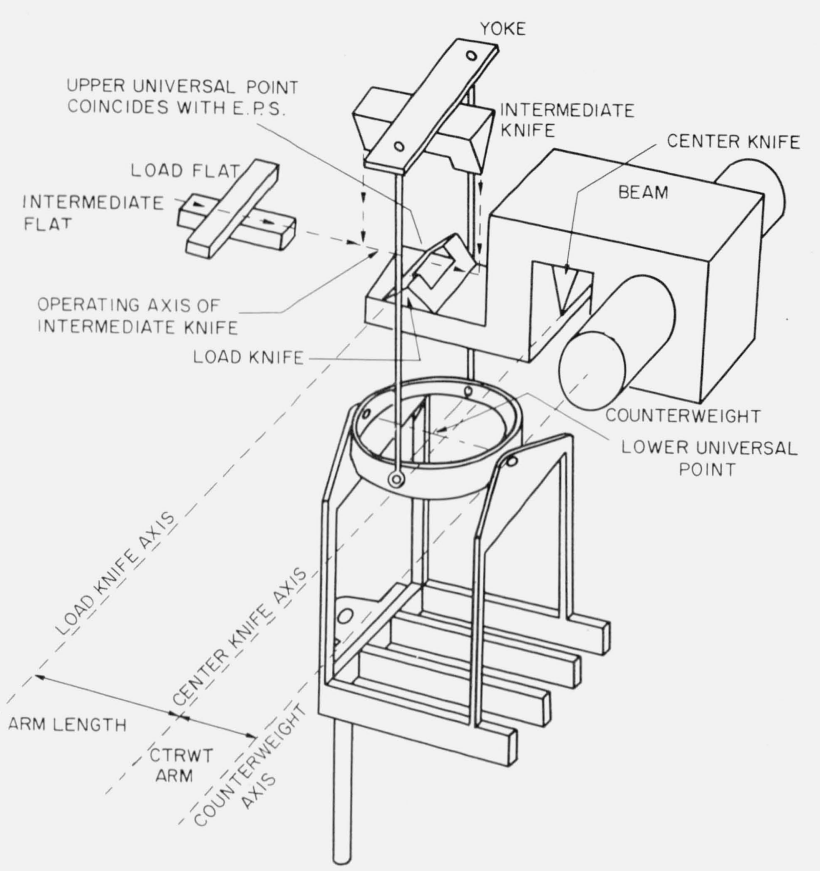

FIGURE 3. An exploded sketch of the oscillating system of the apparatus used in the arrestment study.

For simplicity, the mechanical structure which maintains the load and intermediate flats in their proper relative positions is nct shown. At the top is an exploded view of the knife-edge compensator.

movement of the effective point of suspension arising from rotation of the intermediate knife. A universal coupling or gimbal has been described [2] by which the intermediate knife is always vertically alined (see fig. 4). The major error from nonparallelism of knives then remaining arises from failure of the arrestment system precisely to reengage the suspension with the beam.

\subsection{Change in Inclination of the Terminal Flat}

The exactly analogous error arising from lack of reproducibility of load centering along a line perpendicular to the terminal-knife is associated with the finite knife radius. The error $\delta r$ is illustrated in figure 5 and is of the order of $\rho \delta \alpha$ where $\alpha$ in radians is the inclination of the terminal flat to the horizontal and $\rho$ is the knife-edge radius. Even when $\rho / r$ is less than $10^{-4}, \delta \alpha$ should also be less than $10^{-4}$ in balances working near the highest attainable precision.

Again the universal coupling (fig. 4) assures a highly reproducible inclination of the terminal flat, to the precision required [2], and the major remaining error from inclination of the terminal flat again arises from failure of the arrestment system precisely to reengage the suspension.

\subsection{Nonplanarity of Flats}

Figure 6 will be readily understood as illustrating the effects of nonplanarity of either the center or the terminal flat in conjunction with any failure to reproduce the exact contact configuration between knives and flats. 


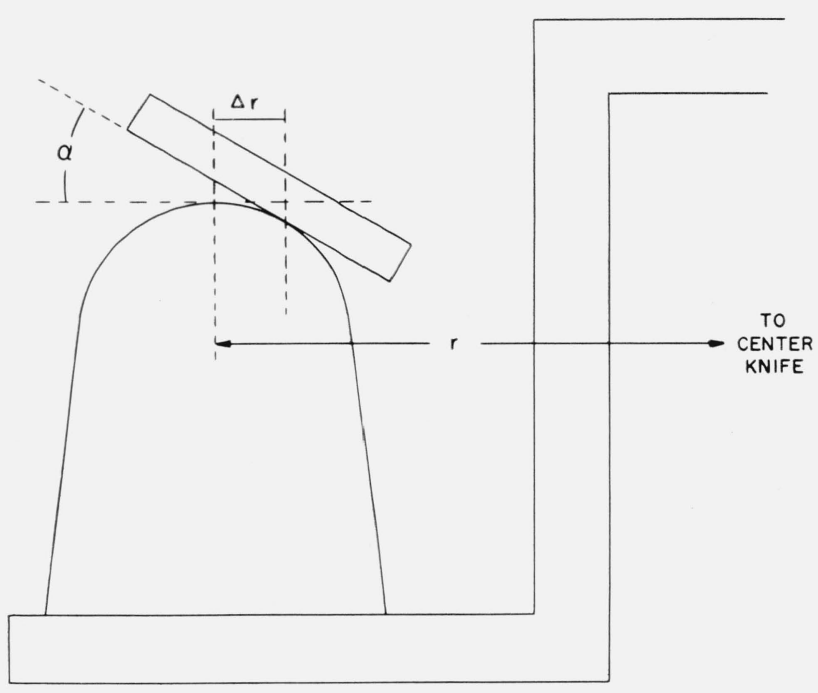

FiguRE 5. If the load flat tilts through an angle $\delta \alpha$, a variation in $\mathrm{r}$ results which is of approximate magnitude $\rho \delta \alpha$.
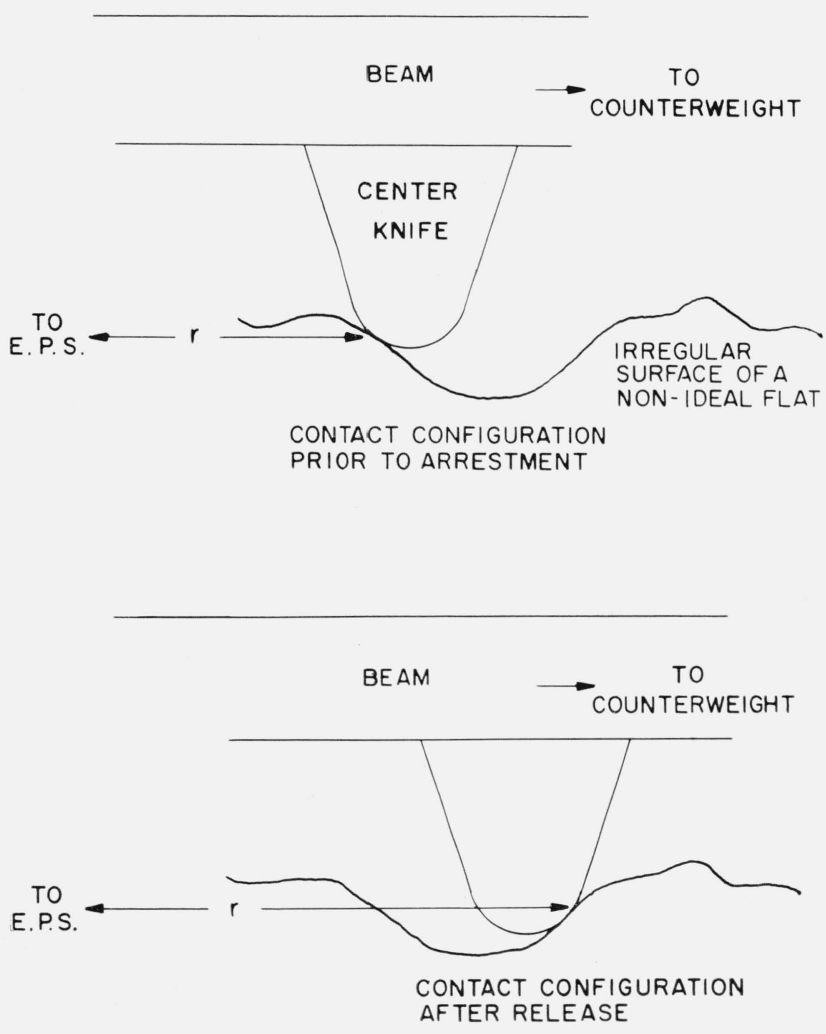

FIGURE 6. This sketch illustrates the variability in $\mathrm{r}$ due to nonreproducible release on a non-ideal flat.

Although the center knife is shown in this sketch, this source of error is present on all knife-flat pairs.

Of course highly sharp knives, and flats that are planar within a fraction of an optical wavelength will help to reduce this error, but it is quite clear that the only good way of eliminating this as well as the two previous errors is not to uncouple knives and flats.
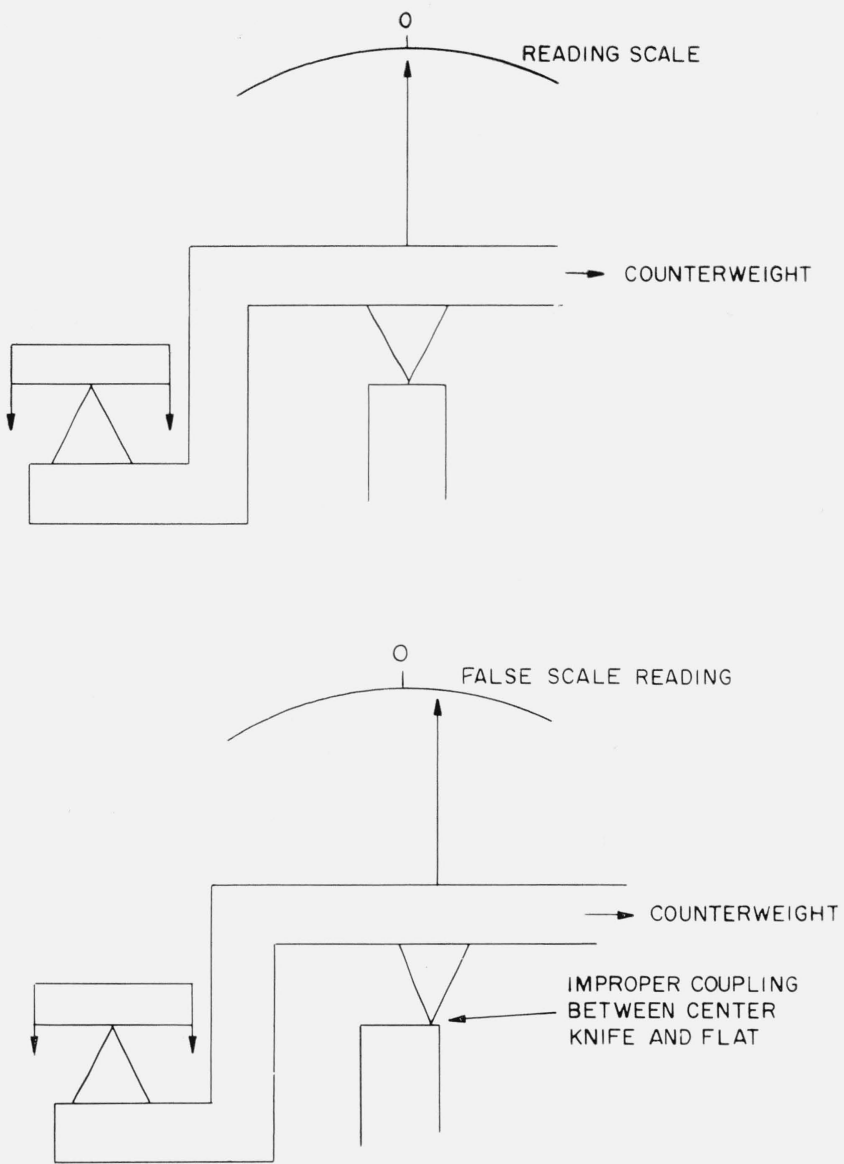

FIGURE 7. This sketch shows a reading error which results from nonreproducible release.

\subsection{Dependence of Balance Indication on Beam Placement}

The error arising from displacement of the pointer relative to the scale or equivalent parts of the read-out system is illustrated in figure 7 . It is another of the errors associated with failure to reproduce the precise contact configuration of the balance bearings but it differs from the others in that it can be eliminated without resorting to a system in which knives are not removed from their flats. The observing system must be sensitive to beam angle alone such as can be attained with an autocollimator.

\subsection{Special Requirement for Arrestment Systems in in Which Bearings Remain Coupled During a Weighing Cycle, "Constant-Load" Arrestment}

Gould [8] after careful study of the one-kilogram balance of the National Physical Laboratory concluded that an improvement in performance would be achieved by retaining center-knife contact. This proved difficult experimentally.

With the simplification associated with a twoknife design we have stipulated not only that both bearings should remain in contact but that the 


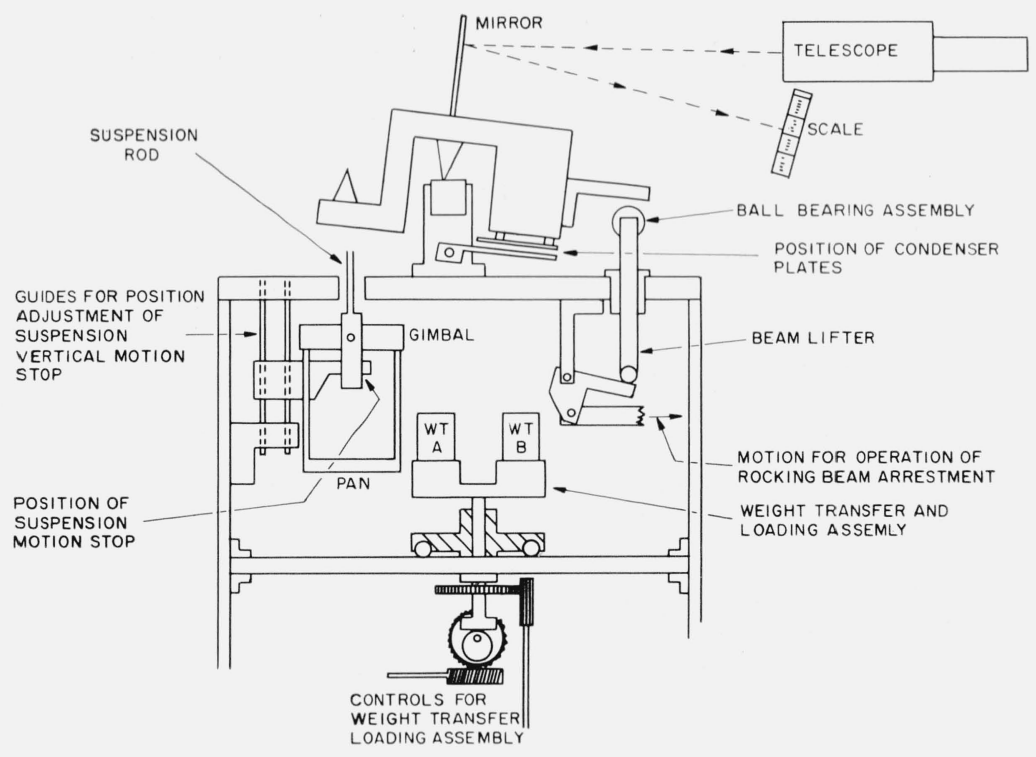

FiguRE 8. Sketch of the apparatus used in the arrestment study.

Instruments installed but not pertinent to this study are eliminated for clarity

knives should be kept loaded, indefinitely, to nearly constant load forces. Our design of constant-load arrestment is described in section 5 .

\subsection{Special Requirements for Arrestment Systems in Which Bearings Are Uncoupled}

It is recognized that situations might exist where decoupling of balance bearings is necessary. Moreover, in our study of sources of arrestment errors an experimental distinction between those specifically connected with the uncoupling operation becomes desirable. It was incumbent upon us to set out the requirements and to design a near optimum arrestment system in which the bearings are uncoupled.

Corwin [6] has pointed out that in practice moving linkages necessarily introduce nonideal movements. We have therefore required an arrestment system to have no moving part responsible for the precision of alinement of the beam or suspension. The alinement should be accomplished by members rigidly and permanently attached to the balance frame.

A study of the history of high-precision balances and the experience of previous workers in the field emphasize the need for the arrestment system to be capable of adjustment by simple rapid procedures.

\section{The Test Instrument}

A flexible test instrument has been constructed. Through the incorporation of thermocouples, vibration pickups, strain gages, displacement gages, etc., it is too complex for routine use as a balance, but rather it is designed for the critical study of systems for-

$$
\begin{aligned}
& \text { a. arrestment } \\
& \text { b. damping } \\
& \text { c. operation in controlled atmospheres }
\end{aligned}
$$

\section{d. readout of balance indication \\ e. vibration control \\ f. temperature control.}

Only the first of these, that is the study of arrestment systems, is described in this paper.

Figure 3 is a sketch of salient members of the oscillating system of the test instrument. Figure 8 shows the external members necessary to the arrestment studies.

\subsection{Bearings}

The $1 \frac{3}{4}$-in. long knives are of Tantung G. The center $\frac{1}{2}$ in. of the terminal knife has been removed to accommodate the intermediate-flat structure. The knife mounting is illustrated in figure 9 . The facets are flat to one fringe and this flatness extends to within 0.0005 in. of the edge as indicated by micro-interferometry.

The agate flats used are up to four fringes high in the center. Whenever electrical contact through the bearings is needed Tantung flats can be used without detectable change in performance.

These are not the best possible bearings. For the arrestment studies it may be an advantage to work with appreciably non-ideal bearings (for example to demonstrate the minimization of the error discussed in section 4.3).

\subsection{The Beam and Suspension}

Only a very few special features deserve mention. The beam [9] differs from the sketch in figure 1 by having a semicylindrical counterweight owing to a last minute decision to reduce the balance capacity to $1 \mathrm{~kg}$. The arm length is 2 inches. In the gimbal [5] of the suspension miniature nonmagnetic ball bearings are used. In the weight-loading system each loading tray can be individually adjusted to match the aspect to the working surface of the pan. 

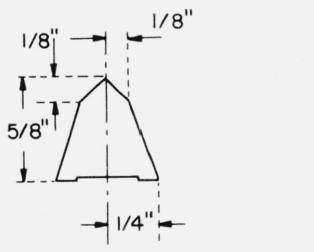

STAINLESS STEEL KNIFE ADJUSTMENT CYLINDER

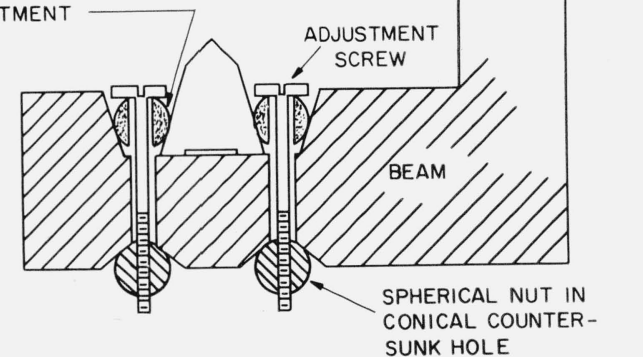

FIGURE 9. The method of mounting and adjusting the knives on the beam.

The center knife is_mounted and adjusted in the same manner.

\subsection{The Arrestment System in Which Bearings Remain Coupled ("Constant-Load" System)}

The mechanical system used for load change without uncoupling the bearings is shown in figure 10 . A spherically ended member is rigidly attached to the upper link of the suspension. Immediately above this member and attached to the frame of the balance is a horizontal flat surface. Both members are stainless steel. Arrestment is accomplished in two steps. First, a force is gradually applied to the system (comparable to the gradual removal of mass from the pan). This causes the suspension to rise slowly until the spherical surface comes into gentle contact with the flat. The force continues to increase; however, further upward suspension motion is prevented by the flat. The increase in force results only in driving these two surfaces into firm contact. When the force is comparable to a 30-mg weight removal, the second step of the process is started. This consists of raising the weight-transfer tray until the weight on the pan has been lifted free and clear. This condition is shown on the right in ifgure 10. When the pan is empty, the force of contact between the sphere and flat is about $1 \mathrm{~kg}$.

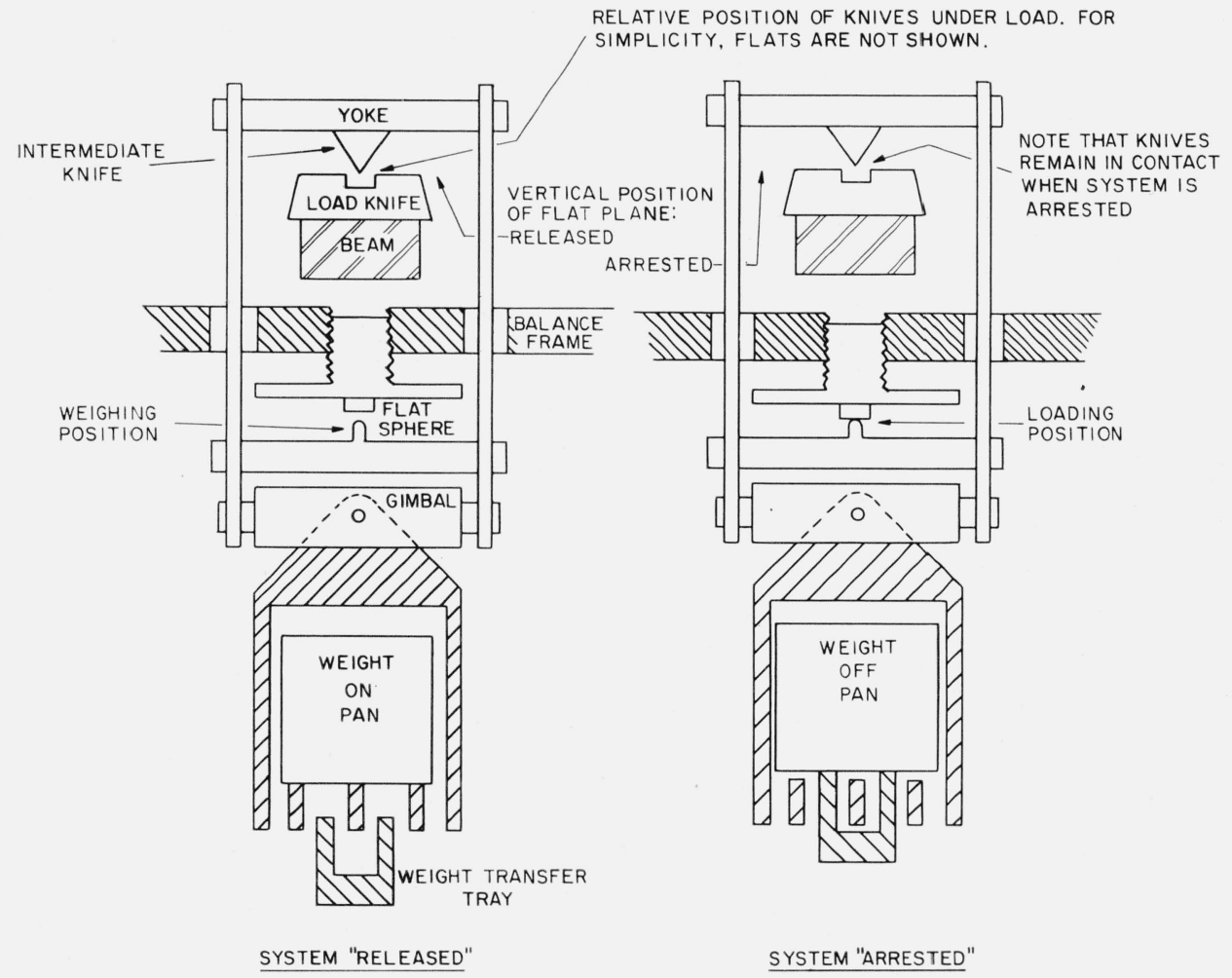

Figure 10. The "constant-load" method of arrestment.

For simplicity the assembly of flats between the load and intermediate knives is not shown-the important point is that these two knives remain in contact with their associated flats in both the "released" condition (on the left) an in the "arrested" condition (shown on the right). To operate the system the weight transfer tray is moved upward. When it comes into contact with the weight on the pan (thereby accepting part of the weight-load from the pan) the suspension moves upward indicating a smaller weight load. When the sphere (on the suspension) touches the flat (on the frame) the upward motion of the suspension is terminated, and continued upward motion of the tray results in lifting the load free and clear of the pan. Under these circumstances, the counterweight moment maintains all knives and flats in the system in contact and loaded at their operating load level. 
The next weight is then lowered onto the pan which returns the contact force between the sphere and flat to its previous value of about $30 \mathrm{mg}$. This force is gradually removed which permits the sphere to fall gently away from the flat and oscillate around its new equilibrium position. The significant point is that during the entire process the contact forces between the knives and flats in the system have varied only slightly from their operating levels. Thus, by maintaining contact between knives and flats during loading, we have minimized knife-flat recoupling errors associated with faulty release. The variable force was applied to the system mechanically by the removal of small weights from hangers on the yoke. It might be mentioned here that we were able to apply a variable force to the beam electrostatically through a condenser arrangement shown in figure 8. This was done in connection with another study not pertinent to arrestment. The data presented in this report were taken when the beam was manipulated by the addition or removal of small weights. The beam can also be manipulated by merely raising the weight transfer tray as shown in figure 10 .

\subsection{The Arrestment System in Which Bearings Are Uncoupled ("Rocking-Beam" System)}

Figure 11 illustrates the essential features of the arrestment system fulfilling the requirements stated in section 4.6. At the top, the balance is shown in its "weighing position." It is rotating about its center knife with the suspension load indicated by the load flat. In the second view the beam lifter has forced the counterweight end of the beam upward and the load end downward until the load flat is "just" in contact with three alinement pins, A, $\mathrm{B}$, and $\mathrm{B}^{\prime}$. Pin $\mathrm{B}^{\prime}$ is on the opposite side of the beam and therefore not visible. These pins are rigidly attached to the bed plate as illustrated. As the beam lifter continues upward, the load flat load is surrendered by the load knife to the three alinement pins. For reasons which will be explained later, the beam lifter has not contributed to the system alinement in this operation. All guidance has been achieved by the regular contact characteristics between the center knife and flat; and, depending upon the geometric perfection of the center knife, the motion of the beam has been around the center knife axis. With the contact broken between the load knife and flat, the beam and suspension are decoupled and the balance is acceptably arrested. Release is accomplished by lowering the beam lifter and permitting the load knife and flat to recontact. If it is desired to break the center-knife contact, the beam is rotated beyond the "partial-arrest" position to the "complete-arrest" position shown at the bottom. The beam-lifter is prevented from placing a horizontal force on the system since it is fabricated from a ball bearing riding on a ball bushing. 'Thus, even though its vertical motion may be imperfect, any horizontal component is taken up by the ball members. This assembly is shown in figure 12 .

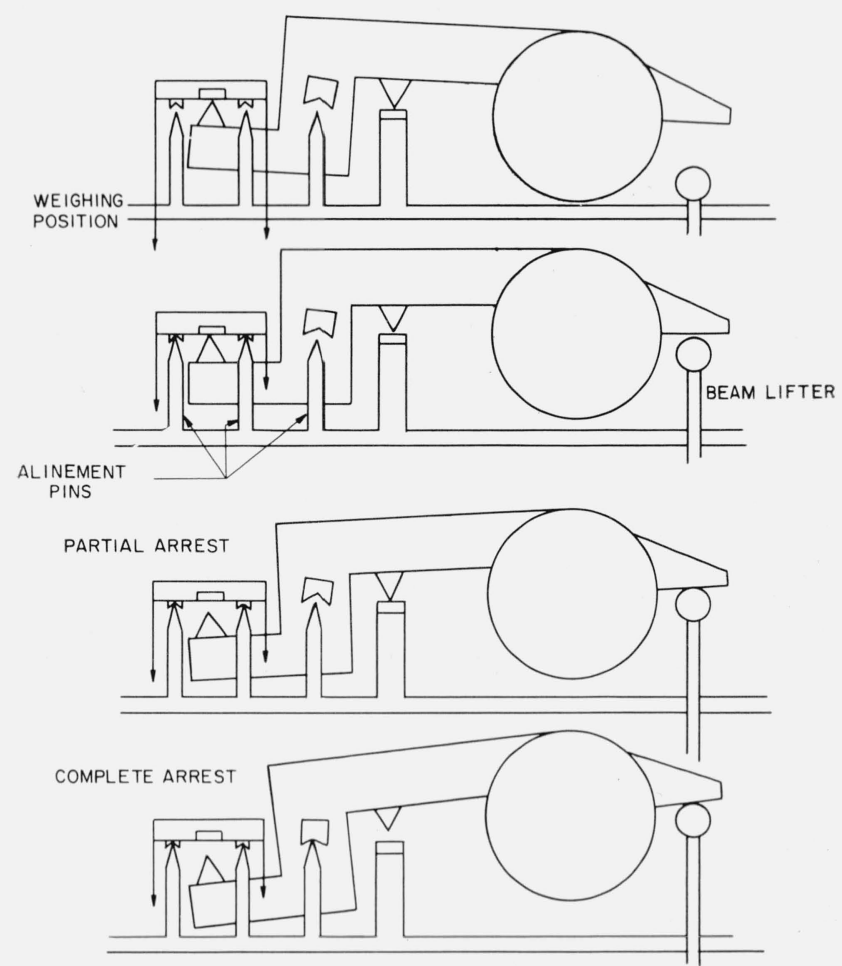

FIGURE 11. The "rocking-beam" method of arrestment.

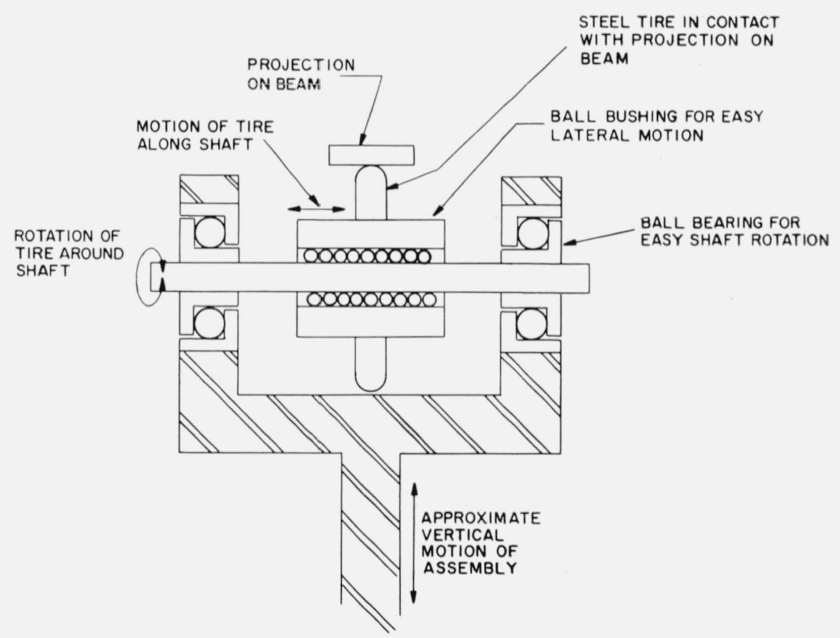

Figure 12. The construction details of the ball bearing device which inhibits the application of horizontal components, to the rocking beam due to imperfect motion of the beam lifter.

\section{The Observed Arrestment Error}

The quantitative evaluation of the arrestment error was accomplished by running the test instrument through many arrestment cycles (as defined in section 3) so that errors associated with slow drift could be effectively eliminated. The error in read-out indication could be demonstrated to be negligible. For the experiments here described the instrument was not artificially damped, and the beam period initially used was 32 sec. 
Apart from the comparison (in section 6.1 below) of the "rocking-beam" arrestment system (compare section 5.4) with the "constant-load" system (compare section 5.3) it was found necessary to investigate (in section 6.2) the influence of the beam-swing period (in section 6.3), the occasional large arrestment errors, and (in section 6.4) the influence of external vibrations.

\subsection{Comparison of "Rocking-Beam" With the "Constant-Load" Arrestment System}

The first test was performed by using the "rockingbeam" arrestment in the "partial-arrest" mode (as described in section 5.4). The same 1-kg brass weight was used to load the pan during each operating cycle. The data taken during this experiment is shown in figure 13A. The standard deviation of the balance indication for one single observation from the estimated mean (shown by the dotted line) is about $15 \mu \mathrm{g}$, that is $1.5 \times 10^{-8}$ of the load. The data indicates that at unpredictable intervals an arrestment error of about $150 \mu \mathrm{g}\left(1.5 \times 10^{-7}\right.$ of the load) can occur (see further discussion in section $6.3)$.

The rest of the test program was conducted on the mode of arrestment in which knives and flats were maintained in contact under constant load throughout the operating cycle (see description in section 5.3). Several evaluation runs were made - a typical set of data is shown in figure 13B. The standard deviation in this run is about $5 \mu \mathrm{g}\left(5 \times 10^{-9}\right.$ of the load) which does not differ significantly from the standard deviation of $4.8 \mu \mathrm{g}$ based on 80 points taken in several similar experiments. As predicted in section 4 the "constant-load" arrestment is significantly superior to the "rocking-beam" system.

\subsection{The Influence of Beam-Swing Period on Arrestment Errors}

When by sensitivity adjustment the beam-swing period was increased to $65 \mathrm{sec}$, the standard deviation associated with the arrestment error apparently increased (fig. 14, curve A).

A closer inspection of the experimental points, however, suggests that the error associated with one of the arrestment cycles is abnormally high. If one concludes that the disturbance to the balance during this cycle was unusually large and that it should therefore be rejected as unrepresentative, one can reinterpret the same data as in curve B in figure 14 . The standard deviation is now about $3 \mu \mathrm{g}$, that is slightly better than for the shorter beam-swing period.

\subsection{The Occasional Large Arrestment Error}

For all the runs so far described it soon became obvious that occasional very large arrestment errors were typical. These could not just be explained by a statistical spread of random results; nor could they be predicted by any irregularities in balance handling. It may be mentioned in passing that a

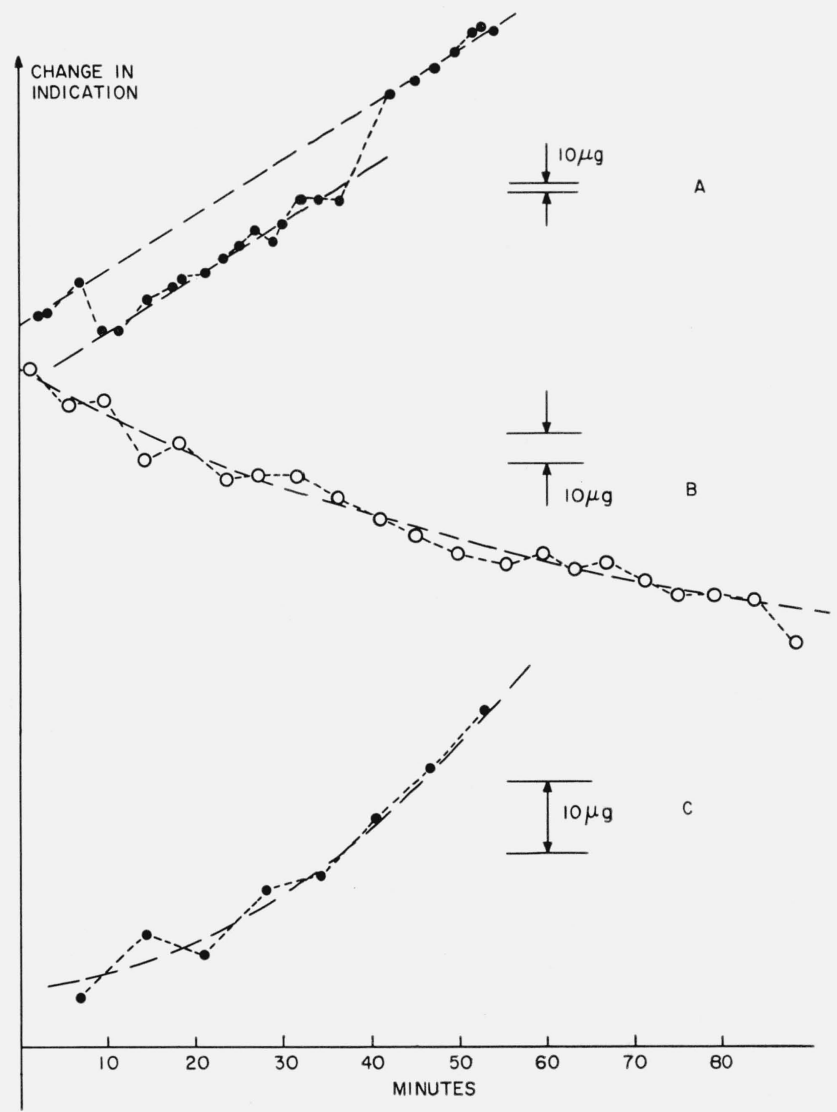

FIgure 13A. Variations in the indicated change in mass, plotted as a function of time when run through 24 operating cycles (defined in the text of the report) using the rocking beam method of arrestment.

B. Variations in the indicated change in mass, plotted as a function of time when run through 24 operating cycles using the constant load method of arrestment.

In this experiment the frequency of the beam system as $1 / 32 \mathrm{c} / \mathrm{s}$.

C. Variations in data when the instrument is operated at $1 / 65 \mathrm{c} / \mathrm{s}$ in a low vibration ambient.

possibly similar effect is associated with the operation of the NPL balance [8], where occasional observations are rejected for presumed mishandling of an arrestment system in which skill is known to play a considerable part.

\subsection{The Influence of External Vibrations}

The occasional unpredictable jump in balance indication became of great importance to this study of arrestment errors. Rejection of some observations is properly resisted by experimenters. Without such rejection the observed arrestment error and ultimate weighing precision is appreciably affected by these occasional unpredictable jumps in balance indication.

It was ultimately discovered that when all vibrational disturbances (nearby ventilation motors and air-conditioning compressors) were removed the occasional large shifts in indication did no longer occur. The observed arrestment error was reduced from 5 to about $3 \mu \mathrm{g}$ (see fig. 13C). 


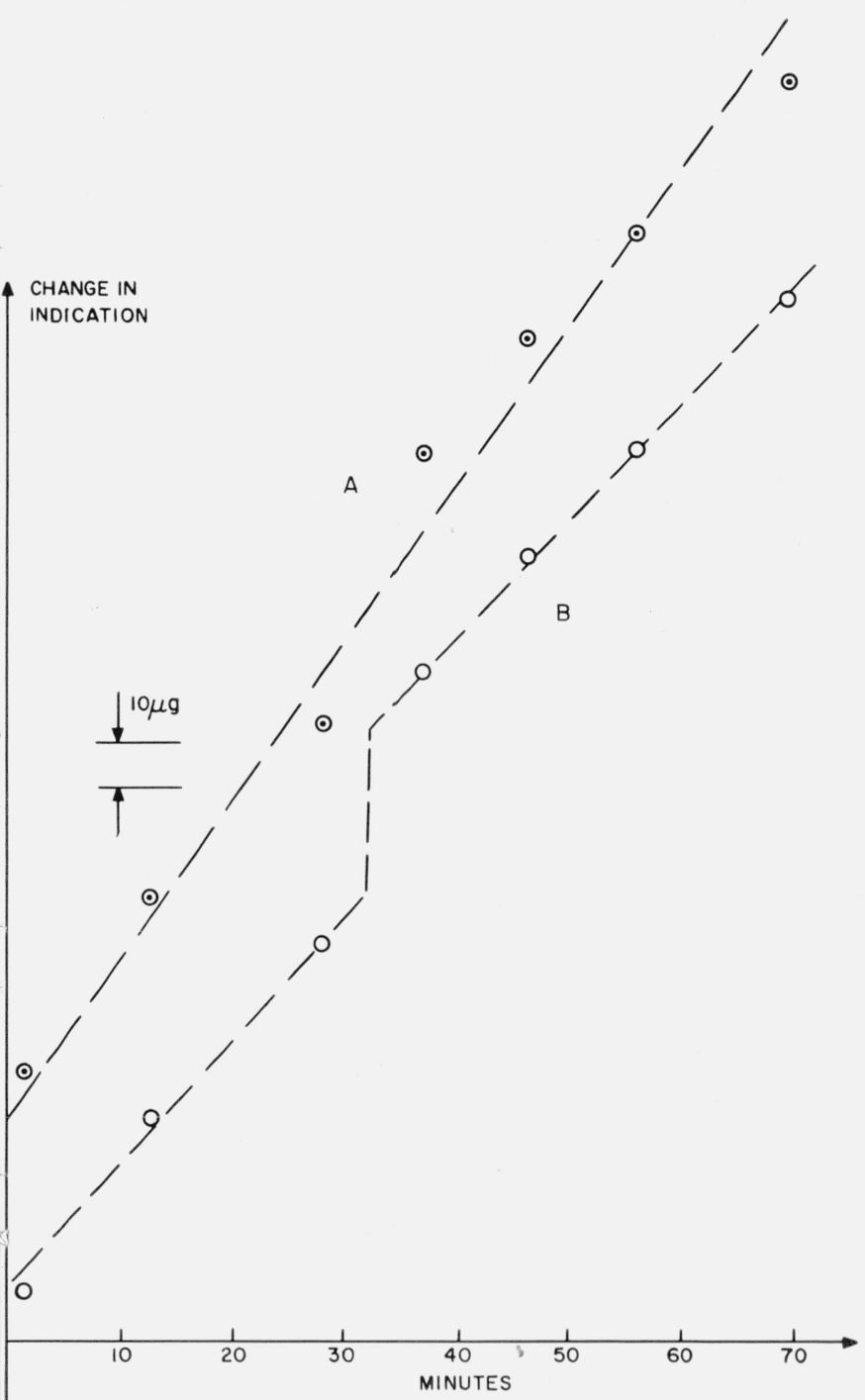

Figure 14A. Variations in indicated change in mass during seven operating cycles.

Balance frequency was $1 / 65 \mathrm{c} / \mathrm{s}$.

B. This is the same data as was presented in figure $14 \mathrm{~A}$ but with most of the data spread forced into one major shift.
The authors wish to express appreciation of the generous assistance and guidance received from many members of the NBS staff during the conduct of this study. L. B. Macurdy, J. M. Frankland, D. P. Johnson very graciously contributed both practical and theoretical assistance. We are especially indebted to $\mathrm{H}$. S. Peiser for continual encouragement and support throughout this work, and for help in the preparation of the manuscript.

Richard Smith, instrument maker, contributed many practical ideas used in the construction of the apparatus. He was frequently called upon to devise an assembly to perform a particular function with no guidance in the mechanical design. Without his valued assistance it would have been difficult indeed to have conducted this study.

Bernard Wasko of the Voland Corporation kindly supplied the knife edges and flats.

\section{References}

[1] H. E. Almer, Response of microchemical balances to change in relative humidity, J. Research NBS $\mathbf{6 4 C}$, $281(1960)$.

[2] H. A. Bowman and L. B. Macurdy, Gimbal device to minimize the effects of off-center loading on balance pans, J. Research NBS 64C, 277 (1960).

[3] H. E. Almer, L. B. Macurdy, H. S. Peiser, and E. A. Weck, Weight calibration schemes for two-knife-edge direct reading balances, J. Research NBS 66C, 33 (1961).

[4] H. S. Peiser, State mass standards, report of the 45th National Conference of Weights and Measures, June 8, 1960 ; p. 45.

[5] T. W. Lashof and L. B. Macurdy, Testing a quickweighing balance, Anal. Chem. 26, 707 (1954).

[6] A. H. Corwin, Weighing technique of organic chemistry Vol. I, Physical Methods of Organic Chemistry 3d Ed., pt. I, ch. III (Interscience Publishers, Inc., New York, 1959).

[7] M. Theissen, Trav. et Mem. Bureau International Pois et Meas., 5, pt. 2 (1886).

[8] F. A. Gould, A knife-edge balance for weighings of the highest accuracy, Proceedings of the Physical Society B 62, 817 (1949).

[9] L. B. Macurdy (private communication).

(Paper 67C3-133) 